\title{
X-RAY MICRO-TOMOGRAPHY CHARACTERIZATION OF VOIDS CAUSED BY THREE-POINT BENDING IN SELECTED ALKALI-ACTIVATED ALUMINOSILICATE COMPOSITE
}

\author{
IVAna Kumpováa ${ }^{a, b, *}$, IVA RozsyPalováa ${ }^{a}$, ZBYNĚK KerŠneR $^{a}$, \\ Pavla RovnaníkOVÁa ${ }^{a}$, Michal VopÁlenskÝ ${ }^{b}$ \\ ${ }^{a}$ Brno University of Technology, Faculty of Civil Engineering, Veveři 331/95, 602 00 Brno, Czech Republic \\ ${ }^{b}$ Czech Academy of Sciences, Institute of Theoretical and Applied Mechanics, Prosecká 809/76, 19000 Prague,
} Czech Republic

* corresponding author: ivana.kumpova@vutbr.cz

\begin{abstract}
This paper deals with the pilot characterization of a special alkali-activated aluminosilicate composite composed of waste brick powder, brick rubble and a solution of potassium water glass. Fracture tests were conducted on the specimens via three-point bending and fracture parameters were evaluated. Selected specimen was investigated using micro-tomography to supplement the results with visual information about the inner structure of this newly designed material before and after the mechanical loading. Tomographic measurements and image processing were conducted for a qualitative and quantitative assessment of changes in the internal structure with an emphasis on the calculation of porosimetric parameters and visualization of the fracture surface. Fractal dimension of fracture surface was estimated.
\end{abstract}

KEYWorDs: Alkali-activated aluminosilicate composite, fracture surface, X-ray micro-tomography, 3D porosity evaluation, fractal dimension.

\section{INTRODUCTION}

A thorough understanding of the initialization process and the formation of cracks and their propagation in various materials is essential in many engineering fields and applications, as one of the crucial safety and economic factors for the preservation of existing and development of new construction [1-3]. Although macro cracks affect the behavior of the structures on a macroscopic scale, critical micro cracking processes leading to structural failure occur in the structure of the material at the microscopic scale. Advanced building materials are commonly characterized only by values of the basic mechanical fracture properties, usually determined by evaluation of quasi-static fracture experiments (e.g. bulk density, modulus of elasticity, effective fracture toughness, effective toughness, and specific fracture energy) and it is difficult to quantify, model and predict their behavior [4 6 .

Current industrial computed tomography $(\mathrm{CT})$ is used to obtain visual information about the state and the spatial arrangement of the internal structure of the investigated material. Using this non-destructive method, the same sample (test specimen) can be scanned repeatedly and observed for changes in its structure occurred as a result of the time-dependent process (maturation, aging, loading with various chemical and physical influences). The method is also perceived as a promising tool to supplement the standard quasi-static fracture experiments with image information related to the spatial distribution of cracks and voids in different phases of failure [7-12]. Re- sults can be used also to validate existing models and predictions.

Advanced digital image processing of tomographically acquired 3D virtual models enables to quantify the representation, spatial orientation and size of individual phases with respect to the achieved resolution. Quantitative description of individual structures and phases in the obtained 3D tomographic model is based on the thresholding of the voxel values and their subsequent registration. It is necessary to realize that the values of voxels forming the $3 \mathrm{D}$ image information is given by a huge range of variables, starting with the parameters of the examined object (e.g. thickness, density, proton number of atoms in individual structures, etc.) through the parameters of the X-ray radiation (e.g. accelerating voltage, efficiency of energy transformation to photon radiation, filtration, etc.) and used detector (e.g. method of the transmitted radiation detection and its conversion to a digital signal, size of pixel matrix, point spread function, sensibility, etc.) to parameters of resulting 3D matrix (e.g. method of reconstruction, image correction, data type, etc.). For this reason, it is generally not possible to determine exactly the threshold for the separation of structures of interest (e.g. voids, fibers, inclusions, etc.) from other present structures. It can be determined more accurately only under the assumptions that comparative measurement of the standard of the material under investigation with known parameters is available. However, this is not common in the case of tomographic research of building materials and thus 
the threshold setting is dependent on the operator's decision. In such case, the results may be affected by a relatively large error and should be checked / paired using other methods.

\section{MATERIAL AND METHODS}

\subsection{SPECIMEN AND MECHANICAL PROPERTIES EVALUATION}

Powdered waste ceramics as precursors for the preparation of alkali-activated aluminosilicate composites have not been much studied in terms of fracture mechanics in the past [13, 14] but they have good prerequisites for future application as environmentally friendly substitutes of cement-based composites. The pilot characterization of special alkali-activated aluminosilicate composite (AAAC) material composed of waste brick powder (grain size range $0-1 \mathrm{~mm}$ ), brick rubble (grain size range $0-4 \mathrm{~mm}$ ) and solution of potassium water glass with silicate modulus $M_{\mathrm{S}}=0-4 \mathrm{~mm}$ was intended. Specimens of nominal dimensions of $40 \times 40 \times 160 \mathrm{~mm}$ ) were provided with an initial edge notch in the middle of the span (the longest dimension) extending to $1 / 3$ of the specimen's depth as seen in Figure 1

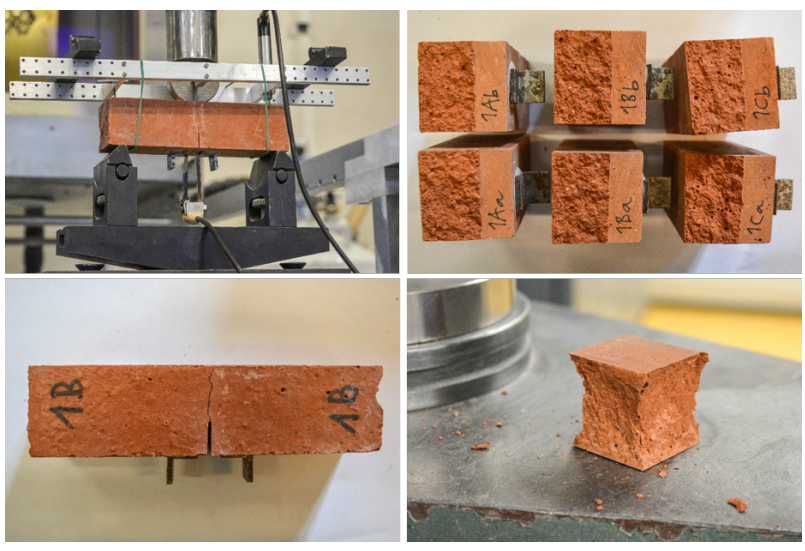

FigURE 1. Illustration of fracture test in three-point bending (left), selected specimens after the fracture tests in three-point bending (top right), and specimen after test in compression (bottom right).

Fracture tests were conducted on specimens via three-point bending according to [15] with a supports span of $140 \mathrm{~mm}$. The 28-days basic mechanical fracture parameters (mean value, coefficient of variation) - bulk density $\left(1911 \mathrm{~kg} \cdot \mathrm{m}^{-3}, 0.9 \%\right)$, modulus of elasticity $(4.64 \mathrm{GPa}, 18.6 \%)$, effective fracture toughness $\left(0.374 \mathrm{MPa} \cdot \mathrm{m}^{\frac{1}{2}}, 7.6 \%\right)$, effective toughness $\left(30.6 \mathrm{~N} \cdot \mathrm{m}^{-1}, 10.1 \%\right)$, and specific fracture energy $\left(45.7 \mathrm{~J} \cdot \mathrm{m}^{-2}, 5.0 \%\right)$ - of the proposed material were obtained from the tests performed on the set of seven test specimens [16]. Fracture parameters were evaluated from load versus deflection diagrams using Effective Crack Model [15] and Work-of-Fracture Method [17]. The informative compressive strength value $(30.6 \mathrm{MPa}, 6.3 \%)$ was determined on the spec- imen's fragments after the fracture tests were completed [18]. Photos in Figure 11illustrate mentioned AAAC specimens, fracture test, as well as test in compression of parts of specimens after three-point bending test. Further mechanical fracture characterization of this AAAC is beyond the scope of this article.

\subsection{X-RAY COMPUTED TOMOGRAPHY}

Before and after the fracture test in three-point bending, one of the specimens was investigated using microtomography to supplement the results with the visual information about the inner structure of the proposed material. Data acquisition for X-ray CT measurements was performed using patented Twinned orthogonal adjustable tomograph (TORATOM, EP 2835631 B1) depicted in Figure 2 The specimen was tomographed by one pair of the X-ray source-detector. The micro-focus X-ray tube (XWT-240-TCHR, X-Ray WorX, Germany) operating at a voltage of $210 \mathrm{kV}$, current on the target of $157 \mu \mathrm{A}$ and a power of $33 \mathrm{~W}$ was used for both CT scans. Gadox flat panel (XRD-1622AP-14, Perkin Elmer, USA) with an active area size of $409.6 \times 409.6 \mathrm{~mm}$, a matrix of $2048 \times 2048 \mathrm{px}$ and a resolution of $200 \mu \mathrm{m}$ per pixel operating at a capacity of $0.5 \mathrm{pF}$ was used as an imaging detector.

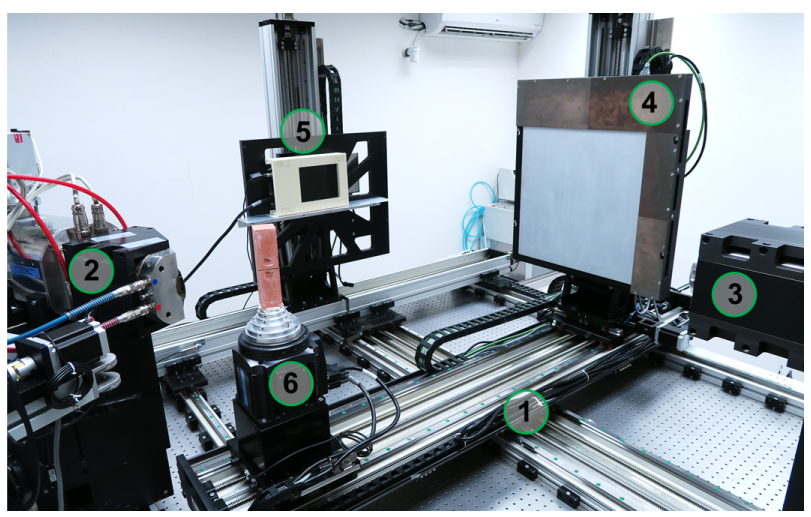

Figure 2. TORATOM tomography system: Active damped anti-vibration table with high-precision CNC positioning system (1) holds two imaging pairs of the $\mathrm{X}$-ray tube $(2,3)$ and detector $(4,5)$ in an orthogonal arrangement with shared rotary stage (6) accommodating the investigated specimen.

By adjusting the assembly to the X-ray spotdetector distance of $1149.90 \mathrm{~mm}$ and the X-ray spotsample distance of $174.26 \mathrm{~mm}$, a geometrical magnification of approximately $6.6 \times$ was achieved, leading to a voxel size (resolution) of $30.3 \mu \mathrm{m}$. The geometrical parameters were chosen in order to obtain the best possible resolution with respect to the size of the detector area and the size of the area of interest on the object being examined, which was determined as a cube with $40 \mathrm{~mm}$ edge centered in the area of the initiation notch. For the correction of acquired projections standard "dark field" and "open beam" (FFC) corrections were used. FFC data was averaged over 100 
images with an acquisition time of $1200 \mathrm{~ms}$. A total of 2880 projections were taken for each tomography with an acquisition time of $1200 \mathrm{~ms}$. The resulting 3D models were calculated by the filtered back projection method using VG Studio Max 3.2 software (Volume Graphics GmbH, Germany).

\subsection{Porosity ANALYSiS}

For the porosity and voids analysis, the threshold algorithm embedded in the Porosity / inclusion analysis module of the VG Studio Max 3.2 software (Volume Graphics GmbH, Germany) was used. Based on the three dimensional analysis of dataset binarized according the selected threshold the algorithm finds all structures below/above this threshold and provides many parameters for each such structure. During the void analysis each voxel is considered a defect if the grey value is below the specified threshold. The threshold was determined by the results of the automatic surface determination procedure based on the histogram. The result is the material boundary defined by one gray value globally applied to the object serving as a threshold: brighter areas are considered as a material, darker areas are considered as a background (air). The surface determination calculates the material boundary in sub-voxel accuracy by trilinear interpolation.

To avoid registration of the noise particles, porosity analysis result was filtered so that only voids bigger than 8 voxels $\left(0.22 \cdot 10^{-3} \mathrm{~mm}^{3}\right)$ were taken into account. According to the number of voids and their volume, partial and total porosity ratio was calculated. For each detected void, the surface area, volume and number of voxels it consists of was monitored. Attention was also paid to sphericity, which is a measure for the ratio between the surface area of a sphere with the same volume as the void and the surface of the void itself.

\subsection{FraCtAL DIMENSION ESTIMATION}

Fracture surface (magistral crack) registered during void analysis was extracted in a form of 3D binary image and its fractal dimension $D$ was estimated by applying the box-counting algorithm embedded in BoneJ plugin of the free open source software ImageJ [19, 20]. In this algorithm - according to the Equation 1 - grids of diminishing size are scanned over the $3 \mathrm{D}$ image and the number of boxes $n$ containing at least one foreground voxel (part of investigated structure) is counted. As the box size $m$ decreases and the grid becomes finer, the proportion of foreground boxes increases in a fractal structure.

$$
D=\frac{\log (m)}{-\log (n)}
$$

The box counting algorithm produces a pair of $\log (m),-\log (n)$ values for each iteration it runs. These pairs are passed to a curve-fitting algorithm, which returns the slope of the linear function which describes them (regression fit). The coefficient of this slope is the fractal dimension value. The box counting algorithm was applied with starting box size of $232 \mathrm{px}$ and box scaling factor of 2. Four grid translations were performed to find the optimal covering.

\section{RESUlts AND Discussion}

The tomographic investigation of the alkali-activated aluminosilicate composite was focused mainly on the determination of the resulting fracture surface from acquired 3D tomographic models. Attention was also paid to the porosimetric parameters or more accurately parameters of voids including both pores and cracks and other defects.

The acquired CT reconstructions were spatially aligned to each other so that the individual tomographic cross sections of the sample before and after loading corresponds. Then the region of interest (ROI) shown in Figure 3 was subsequently selected from both models, large enough not to lose its meaningful value, however not including the areas of the surrounding air and initiating notch, which would interfere with further processing. Dimensions of the selected ROI were $39.82 \times 26.58 \times 39.42 \mathrm{~mm}$.

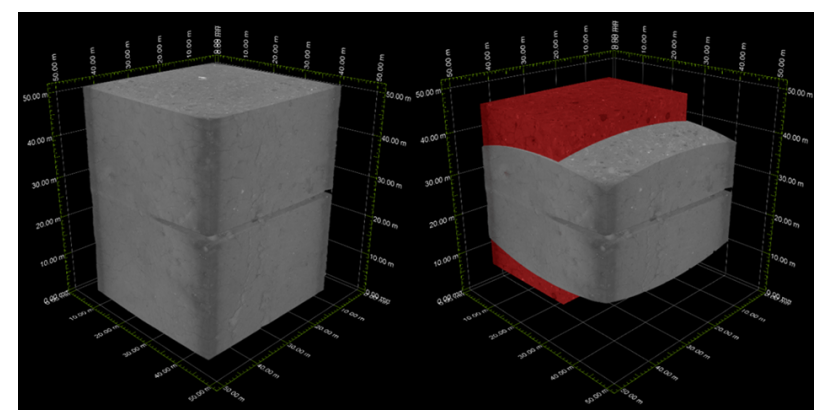

FiguRE 3. Visualization of the obtained 3D model of the scanned area. Part indicated by the red color specifies the volume used for the determination of porosimetric parameters and further investigation of the crack spatial distribution.

A differential model was calculated to visualize and highlight the differences in the internal structure of the specimen caused by fracture. An example cross sections are shown in Figure 6 and Figure 7 . 3D spatial model of the magistral crack registered during void analysis was extracted. From the results shown in Figure 8 the macro-crack fractal dimension of 2.154 was estimated, which is typical value for concrete.

Further selected porosimetric results are summarized in the table in Figure 4 and dependence of number of pores on their size and their contribution to total porosity is graphically expressed in Figure 5 . Several conclusions can be estimated. A newly formed macro crack with a volume of $45.66 \mathrm{~mm}^{3}$ and a surface of $1500 \mathrm{~mm}^{2}$ has been revealed. Its projected length in $\mathrm{X}, \mathrm{Y}, \mathrm{Z}$ directions was estimated to $39.44 \mathrm{~mm}$, $17.91 \mathrm{~mm}$ and $7.05 \mathrm{~mm}$ respectively. Area of the crack 


\begin{tabular}{|c|c|c|c|c|c|c|c|c|c|}
\hline \multicolumn{2}{|c|}{ Pore volume interval } & \multicolumn{4}{|c|}{ ROI before loading, volume $=40279 \mathrm{~mm}^{3}$, porosity $1.497 \%$} & \multicolumn{4}{|c|}{ ROI after loading, volume $=40280 \mathrm{~mm}^{3}$, porosity $1.699 \%$} \\
\hline From $\left[\mathrm{mm}^{3}\right]$ & To $\left[\mathrm{mm}^{3}\right]$ & Pores cnt & Pores vol $\left[\mathrm{mm}^{3}\right]$ & avg Surface $\left[\mathrm{mm}^{2}\right]$ & avg Spheric ity & Pores cnt & Pores vol $\left[\mathrm{mm}^{3}\right]$ & avg Surface $\left[\mathrm{mm}^{2}\right]$ & avg Sphericity \\
\hline 0.0000 & 0.0005 & 48354 & 15.4225 & 0.0342 & 0.6630 & 63955 & 20.2825 & 0.0350 & 0.6448 \\
\hline 0.0005 & 0.0010 & 27275 & 18.8763 & 0.0596 & 0.6386 & 33078 & 22.7312 & 0.0614 & 0.6174 \\
\hline 0.0010 & 0.0020 & 17074 & 23.7321 & 0.0976 & 0.6238 & 20124 & 27.9137 & 0.1015 & 0.6010 \\
\hline 0.0020 & 0.0050 & 12376 & 38.0870 & 0.1708 & 0.6092 & 13792 & 42.2815 & 0.1783 & 0.5858 \\
\hline 0.0050 & 0.0100 & 4641 & 32.5783 & 0.3079 & 0.5948 & 5145 & 35.8996 & 0.3252 & 0.5676 \\
\hline 0.0100 & 0.0200 & 2502 & 34.8843 & 0.5019 & 0.5859 & 2724 & 37.8271 & 0.5340 & 0.5576 \\
\hline 0.0200 & 0.0500 & 1829 & 56.2166 & 0.8699 & 0.5813 & 1893 & 58.0637 & 0.9280 & 0.5525 \\
\hline 0.0500 & 0.1000 & 706 & 49.2620 & 1.5486 & 0.5814 & 753 & 52.6570 & 1.7064 & 0.5450 \\
\hline 0.1000 & 0.2000 & 405 & 56.7852 & 2.5238 & 0.5836 & 401 & 55.8287 & 2.7893 & 0.5436 \\
\hline 0.2000 & 0.5000 & 272 & 84.7035 & 4.6737 & 0.5746 & 289 & 87.7943 & 4.9375 & 0.5330 \\
\hline 0.5000 & 1.0000 & 70 & 48.1447 & 9.7496 & 0.5449 & 77 & 51.7069 & 11.0301 & 0.4870 \\
\hline 1.0000 & 2.0000 & 45 & 60.7502 & 17.2095 & 0.5504 & 42 & 56.3886 & 16.5300 & 0.4969 \\
\hline 2.0000 & 5.0000 & 16 & 53.1381 & 21.7970 & 0.5656 & 17 & 56.7670 & 38.8571 & 0.4294 \\
\hline 5.0000 & 10.0000 & 3 & 15.9530 & 22.9930 & 0.6400 & 3 & 17.4295 & 74.1824 & 0.4167 \\
\hline 10.0000 & 20.0000 & 1 & 14.4500 & 45.7704 & 0.6300 & 1 & 15.0080 & 61.7993 & 0.4800 \\
\hline 20.0000 & 50.0000 & 0 & & & & 1 & 45.6560 & 1500.0000 & 0.0400 \\
\hline
\end{tabular}

FiguRE 4. Table summarizing selected porosimetric parameters.

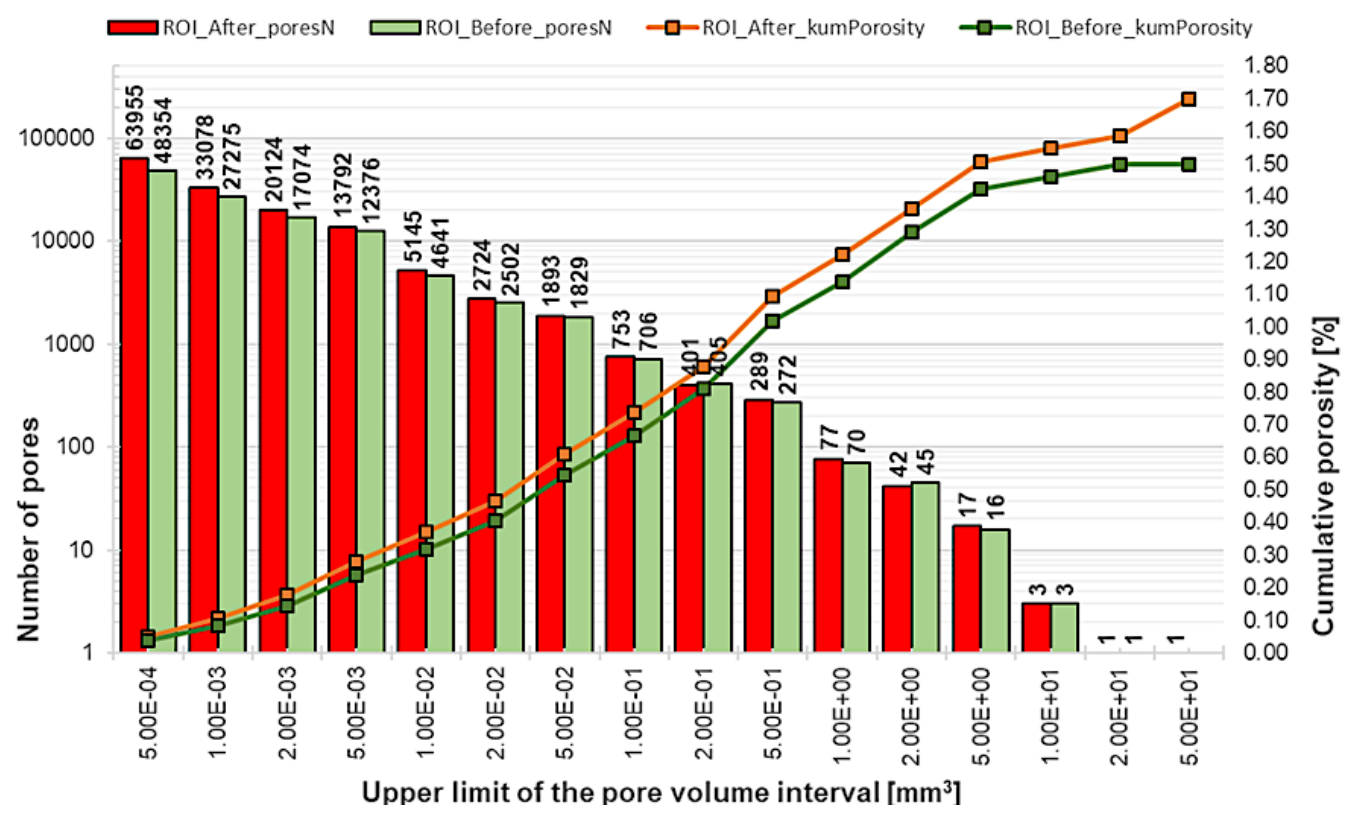

FIGURE 5. Graph of the porosimetric results. Bars in the graph represent histogram of the pore distribution within the investigated ROI before and after the three-point bending fracture test. Curves on the secondary axis represent the cumulative porosity at each pore volume interval.

projected in $x y, x z, y z$ plane (for orientation see Figure 6 and Figure 7 was calculated to $320.00 \mathrm{~mm}^{2}$, $82.06 \mathrm{~mm}^{2}$ and $41.55 \mathrm{~mm}^{2}$ respectively.

After the loading, the formation of new voids and the increase of the volume of the existing ones can be observed, which is caused by the thinning and cracking of the material. Total porosity of the ROI after loading increased by $0.2 \%$ with the macro crack contribution of $0.11 \%$. An increase of the average void's surface and decrease of the average sphericity ratio indicates the formation of the micro cracks. In the case of material with compact not interconnected pores, as AAAC presented, these values can serve very well to separate the results of the void analysis on pores (high sphericity) and cracks (low sphericity) and further investigation of one group only.

\section{Conclusions}

Powdered waste ceramics as precursors for the preparation of selected alkali-activated aluminosilicate composite were studied in this paper. After the previously determined mechanical fracture parameters of this material, attention was focused on the use of advanced tomographic method to analyze the porosity and to determine the fractal dimension of the projected fracture surface of selected specimen after fracture test in three-point bending.

\section{ACKNOWLEDGEMENTS}

This outcome has been achieved within the framework of the project of the specific university research of the Brno University of Technology No. FAST-J-19-6084 and with the financial support of the Czech Science Foundation un- 


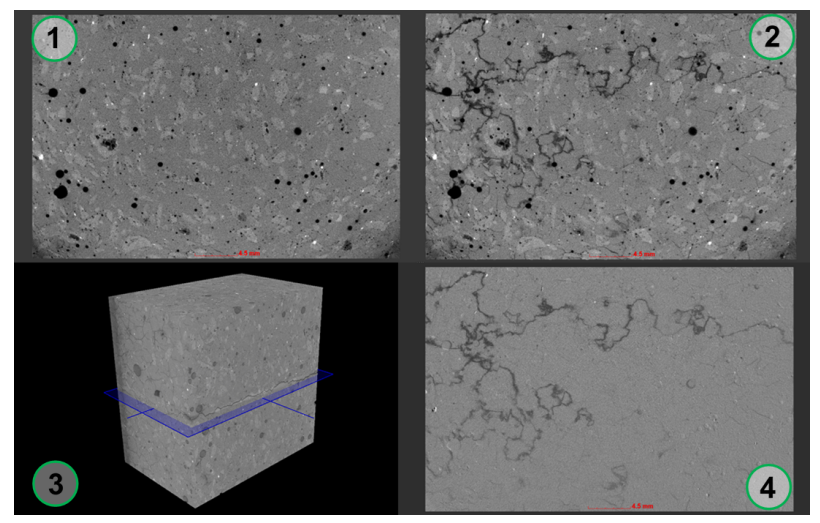

FIGURE 6. Visualization of tomographic cross-sections taken in the same place parallel to the direction of the crack propagation ( $x y$ plane). 1) Visualization of the cross-section before three-point bending; 2) Visualization of the cross-section after three point bending; 3) Plane representing the position of the visualized cross-sections (orientation of the volume corresponds to Figure 3); 4) Differential image with highlighted differences (cracks).
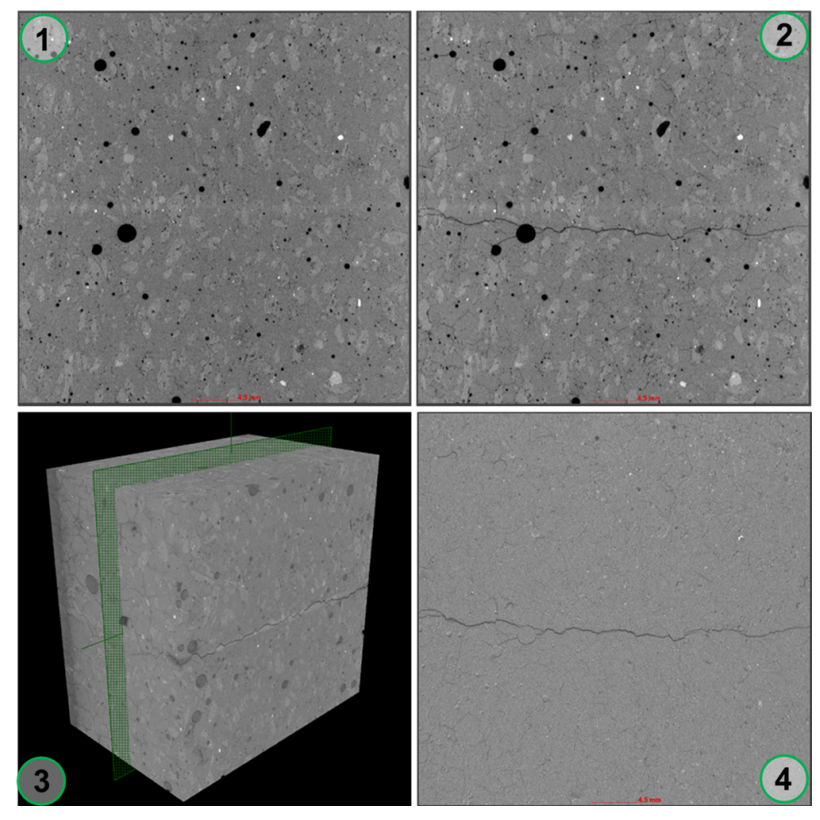

FiguRE 7. Visualization of tomographic cross-sections taken in the same place perpendicular to the direction of the crack propagation ( $x z$ plane). 1) Visualization of the cross-section before three-point bending; 2) Visualization of the cross-section after three point bending; 3) Plane representing the position of the visualized cross-sections (orientation of the volume corresponds to Figure 3); 4) Differential image with highlighted differences (cracks).

der project No 19-01982S Alkali-activated aluminosilicate composites based on ceramic precursors.

\section{REFERENCES}

[1] K. Germaniuk, T. Gajda, A. Sakowski, et al. Bridge structures cracks-what made that phenomena so common? Transportation Research Procedia 14:4030-4039, 2016. DOI:10.1016/j.trpro.2016.05.500

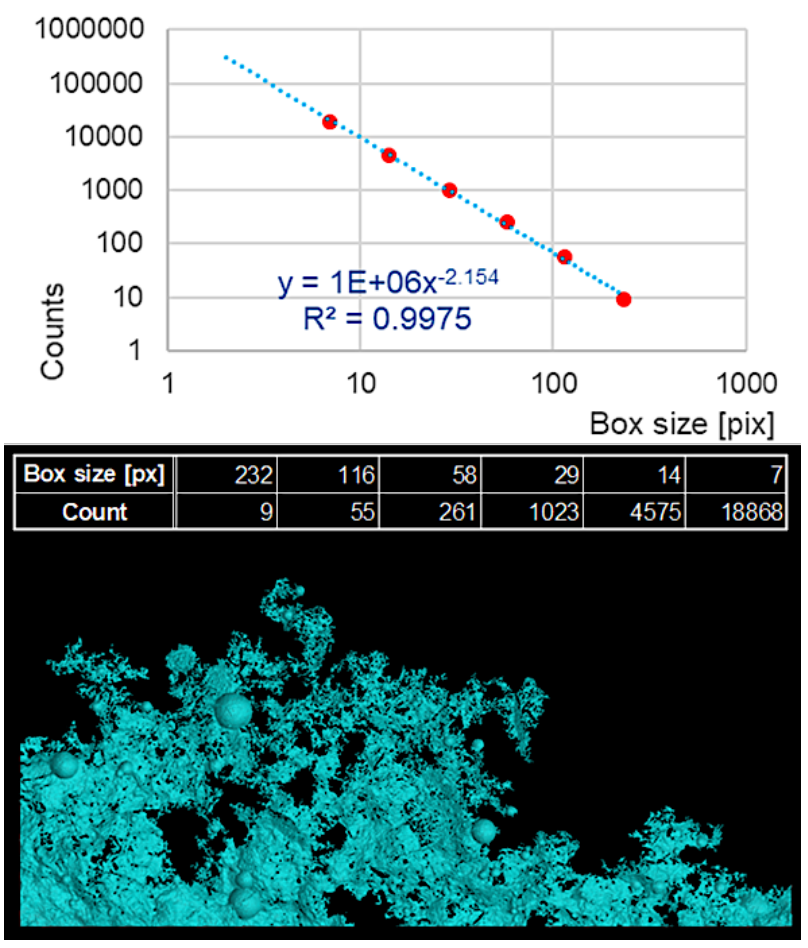

Figure 8. Visualization of the extracted fracture surface with the table and graph of the values obtained using box counting algorithm.

[2] Q. W. Ren, Q. Li, S. Liu. Research advance in failure risk and local strength failure for high arch dams. Chinese Science Bulletin 57(36):4672-4682, 2012. DOI:10.1007/s11434-012-5563-7.

[3] S. Xu, H. W. Reinhardt. Crack extension resistance and fracture properties of quasi-brittle softening materials like concrete based on the complete process of fracture. International Journal of Fracture 92(1):71-99, 1998. DOI:10.1023/A:1007553012684

[4] J. Schijve. Fatigue of structures and materials. 2nd. ed. Springer, Netherlands, 2009. DOI:10.1007/978-1-4020-6808-9.

[5] H. Schorn, M. Butler. Microcrack propagation observed in ESEM. In Proceedings of the 2002 SEM Annual Conference and Exposition on Experimental and Applied Mechanics, p. 4. Society for Experimental Mechanics, Wisconsin USA, 2002.

[6] U. Krupp. Fatigue Crack Propagation in Metals and Alloys: Microstructural Aspects and Modelling Concepts. WILEY-VCH Verlag GmbH \& Co. KGaA, Weinheim, 2007. DOI:10.1002/9783527610686.

[7] L. Skarzynski, J. Tejchman. Experimental investigations of fracture process in concrete by means of x-ray micro-computed tomography. Strain 52(1):26-45, 2016. DOI:10.1111/str.12168.

[8] W. Trawinski, J. Bobinski, T. J. Two-dimensional simulations of concrete fracture at an aggregate level with cohesive elements based on x-ray $\mu \mathrm{CT}$ images. Engineering Fracture Mechanics 168:204-226, 2016. DOI:10.1016/ j.engfracmech.2016.09.012.

[9] L. Vavro, K. Soucek, D. Kytyr, et al. Visualization of the evolution of the fracture process zone in sandstone 
by transmission computed radiography. vol. 191, pp. 689-696. 2017. DOI:10.1016/j.proeng.2017.05.233

[10] I. Jandejsek, F. Nachtrab, N. Uhlmann, D. Vavrik. $\mathrm{X}$-ray dynamic defectoscopy utilizing digital image correlation. Nuclear Instruments and Methods in Physics Research Section A: Accelerators, Spectrometers, Detectors and Associated Equipment 633:185-186, 2011. DOI:10.1016/j.nima.2010.06.162

[11] I. Kumpova, T. Fila, D. Vavrik, Z. Kersner. X-ray dynamic observation of the evolution of the fracture process zone in a quasi-brittle specimen. Journal of Instrumentation 10(8):C08004, 2015. DOI:10.1088/1748-0221/10/08/C08004.

[12] I. Kumpova, M. Vopalensky, T. Fila, et al. On-the-fly fast x-ray tomography using a cdte pixelated detector application in mechanical testing. IEEE Transactions on Nuclear Science 65(12):2870-2876, 2018. DOI:10.1109/TNS.2018.2873830

[13] P. Chindaprasirt, P. Paisitsrisawat, U. Rattanasak. Strength and resistance to sulfate and sulfuric acid of ground fluidized bed combustion fly ash-silica fume alkali-activated composite. Advanced Powder Technology 25(3):1087-1093, 2014. DOI:10.1016/j.apt.2014.02.007

[14] D. Bondar, C. J. Lynsdale, N. B. Milestone, et al. Effect of type, form, and dosage of activators on strength of alkali-activated natural pozzolans. Cement and Concrete Composites 33(2):251-260, 2011. DOI:10.1016/j.cemconcomp.2010.10.021

[15] B. L. Karihaloo. Fracture Mechanics and Structural Concrete. Longman Scientific \& Technical, 1995. ISBN: 978-0582215825.
[16] I. Rozsypalova, I. Kumpova, T. Majda, et al. Basic mechanical fracture characterization of a selected alkali-activated aluminosilicate composite. In EUROMAT 2019 Abstracts, p. 2157. Swedish Society for Materials Technology, 2019.

[17] RILEM TC FMC 1: Determination of the fracture energy of mortar and concrete by means of three-point bend tests on notched beams. Materials and Structures 18(4):287-290, 1985. DOI:10.1007/BF02472918

[18] British Standards Institution. EN 13892-2:2002 (E): Methods of test for screed materials-Part 2:

Determination of flexural and compressive strength, 2002.

[19] M. Doube, M. M. Kłosowski, I. Arganda-Carreras, et al. Bonej: Free and extensible bone image analysis in imagej. Bone 47(6):1076-1079, 2010. DOI:10.1016/j.bone.2010.08.023

[20] N. L. Fazzalari, I. H. Parkinson. Fractal dimension and architecture of trabecular bone. The Journal of Pathology 178:100-105, 1996. DOI:10.1002/(SICI)10969896(199601)178:1<100::AID-PATH429>3.0.CO;2-K. 